\title{
Электронный парамагнитный резонанс электронов проводимости в кристаллах $\mathrm{HgSe}$
}

\author{
() А.И. Вейнгер ${ }^{1}$, И.В. Кочман ${ }^{1, \uparrow}$, В.И. Окулов ${ }^{2}$, М.Д. Андрийчук ${ }^{3}$, Л.Д. Паранчич ${ }^{3}$ \\ ${ }^{1}$ Физико-технический институт им. А.Ф. Иоффе Российской академии наук, \\ 194021 Санкт-Петербург, Россия \\ ${ }^{2}$ Институт ффизики металлов им. М.Н. Михеева Уральского отделения Российской академии наук, \\ 620137 Екатеринбург, Россия \\ 3 Черновицкий национальный университет, \\ 58012 Черновцы, Украина \\ ๑ E-mail: kochman@mail.ioffe.ru
}

(Получена 4 мая 2018 г. Принята к печати 21 мая 2018 г.)

Изучены образцы бесщелевого полупроводника $\mathrm{HgSe}$ с различной концентрацией примеси железа. Образцы $\mathrm{HgSe}: \mathrm{Fe}$ исследованы методом электронного парамагнитного резонанса. Рассмотрены множественные резонансные линии, обусловленные неспаренными спинами различного происхождения. Для описания свойств электронов, локализованных на мелких примесях, использована водородоподобная модель. Рассмотрено влияние внутреннего поля на резонансные линии. Обнаружено, что зона проводимости $\mathrm{HgSe}$ является не только непараболической, но также и несферической.

DOI: 10.21883/FTP.2018.13.46868.8904

\section{1. Введение}

Электронный парамагнитный резонанс (ЭПР) в настоящее время является одним из широко применяемых методов исследования твердого тела. Из анализа его спектров получают богатую информацию о магнитных моментах как невзаимодействующих, так и взаимодействующих ионов, а также о ближайшем окружении парамагнитного иона. При этом используют различные подходы для описания резонансных спектров в различных твердых телах: в ионных кристаллах, в полупроводниках, в полимерах, в жидкостях и т.д. Наша работа посвящена исследованию ЭПР электронов в зоне проводимости в кристаллах узкозонного полупроводника $\mathrm{HgSe}$. Для анализа спектров ЭПР использован подход, обычно применяемый для описания мелких примесных центров в полупроводниках [1].

Как известно [2], электроны, локализованные на мелких примесных центрах, обладают некомпенсированными спинами, которые также способны проявляться в ЭПР. Для описания магнитных свойств таких электронов используют водородоподобную модель. Примесный атом представляется как единое целое, и только один электрон, слабо связанный с атомом, обладает некомпенсированным спином с $S=1 / 2$. В этом случае $g$-фактор достаточно простыми соотношениями связан с величиной эффективной массы. Для эллипсоидальной изоэнергетической поверхности

$$
g_{\|}=2-\left(\delta / E_{13}\right)\left(m_{0} / m_{t}-1\right)
$$

и

$$
g_{\perp}=2-\left(\delta / E_{13}\right)\left(m_{0} / m_{l}-1\right),
$$

где $g_{\|}$и $g_{\perp}-$ главные значения $g$-фактора, $\delta$ параметр спин-орбитального расщепления, $E_{13}-$ энер- гетическая щель между валентной зоной и зоной проводимости при $k=0$ (центр зоны Бриллюэна), $m_{t}$ и $m_{l}-$ значения эффективной массы электрона в направлениях главных осей эллипсоида эффективных масс.

В вырожденном полупроводнике, когда уровень Ферми находится в зоне проводимости, величина эффективной массы в первом приближении сохраняется и, следовательно, величина $g$-фактора должна оставаться приблизительно такой же, как для мелкой примеси. Из формул $(1 a)$ и (16) видно, что малой эффективной массе соответствует большая отрицательная величина $g$-фактора. Экспериментальные значения этого параметра были получены из анализа осцилляций Шубникова-де Гааза (ШдГ) [3]: $g \approx-13--8.6$. Это значит, что линии ЭПР спектра для электронов в зоне проводимости следует искать в слабых полях $[4,5]$.

В настоящей работе приведены результаты наблюдения таких спектров. Обсуждаются их особенности, которые, по-видимому, связаны с большим временем жизни спинов в виртуальных состояниях и малой глубиной скин-слоя. Сделаны выводы о взаимодействии спинов в зоне проводимости с решеткой кристаллов $\mathrm{HgSe}$, легированных железом с концентрацией примеси $\sim 7 \cdot 10^{18} \mathrm{~cm}^{-3}$.

\section{2. Образцы, методика измерений и обработка результатов}

Измерения проводились на трех образцах $\mathrm{HgSe}$, изготовленных в Черновицком национальном университете (Украина). Они были легированы $\mathrm{Fe}$, концентрация которого составляла $7 \cdot 10^{18}, 1 \cdot 10^{19}$ и $1 \cdot 10^{21} \mathrm{~cm}^{-3}$. Это соответствует изменению удельного сопротивления в диапазоне $(1-3) \cdot 10^{-5}$ Ом · см [6]. Размеры образцов 
составляли $10 \times 2 \times 1$ мм. Электрофизические характеристики образцов $\mathrm{HgSe}$ более подробно изложены в [7].

В образцах с таким низким сопротивлением микроволновое поле, которое поглощается при резонансе, не проникает на всю глубину образца. Его проникновение ограничивается глубиной скин-слоя $\delta$. Оценки глубины скин-слоя по стандартным формулам показывают, что в этом случае $\delta=(1.5-3) \cdot 10^{-3}$ мм, т. е. около 1 мкм. Таким образом, микроволновое поле целиком сосредоточено вблизи поверхности образца. Это приводит к искажению линий резонансного поглощения. Такое искажение делает невозможным определение концентрации спинов стандартным способом: путем двойного интегрирования спектра производной линии поглощения [8].

Для исследований мы использовали ЭПР спектрометр E-112 фирмы „VARIAN“ с проточным гелиевым криостатом „Oxford Instruments ESR-910“, который способен поддерживать температуру образца в широком диапазоне температур $(2-300 \mathrm{~K})$. Измерения проводились по классической ЭПР методике. Единственная сложность при измерениях состояла в том, что сопротивление образца $R$ следовало поддерживать существенно большим или существенно меньшим, чем приведенное сопротивление волновода $\rho$, поскольку при $R \approx \rho$ чувствительность спектрометра заметно снижается [9]. Следует отметить, что спектр ЭПР, приписываемый электронам в зоне проводимости, наблюдается только в наименее легированном образце. В двух более сильно легированных образцах такой спектр не наблюдался.

\section{3. Результаты эксперимента}

Вид участка спектра ЭПР образца $\mathrm{HgSe}$ с концентрацией железа $N_{\mathrm{Fe}}=7 \cdot 10^{18} \mathrm{~cm}^{-3}$ показан на рис. 1 в магнитном поле $0<H<2.8$ кЭ. Магнитное поле

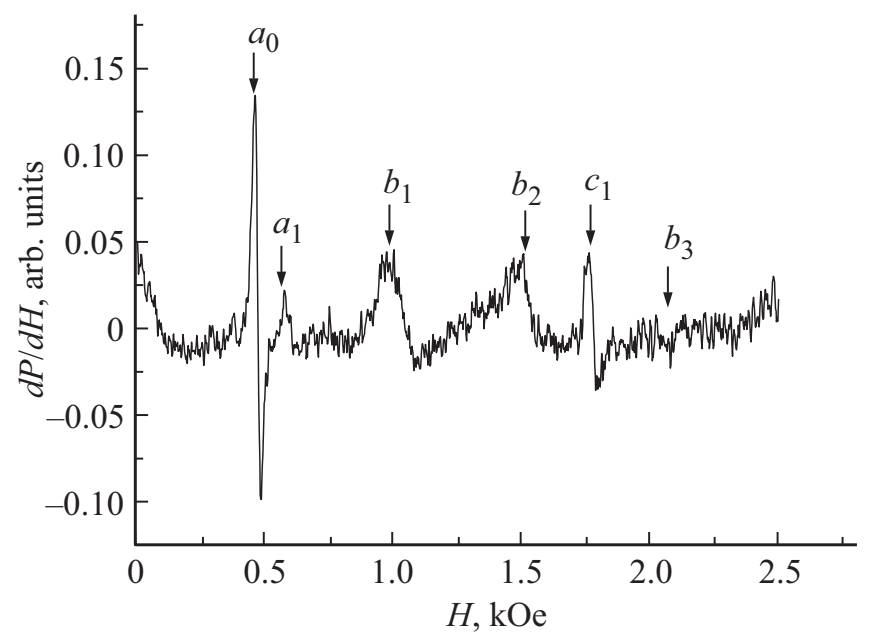

Рис. 1. Вид спектра ЭПР образца $\mathrm{HgSe}: \mathrm{Fe}$ с концентрацией $N_{\mathrm{Fe}}=7 \cdot 10^{18} \mathrm{~cm}^{-3}$ при направлении магнитного поля вдоль оси [001]. Стрелки указывают на предположительно идентифицированные линии. Объяснения в тексте.
$H \|$ [001], температура $T=3 \mathrm{~K}$. В более сильных полях около 3.5 кЭ наблюдаются спектры ЭПР от примесных ионов $\mathrm{Fe}^{3+}$, описанные ранее в [10]. При дальнейшем увеличении магнитного поля начинают проявляться осцилляции магнитосопротивления (осцилляции Шубникова-де Гааза), которые исследованы в кристаллах $\mathrm{HgSe}$ в ряде работ [11]. В связи с этим в настоящем статье мы ограничимся рассмотрением той части спектра, который представлен на рис. 1 .

Резонансные линии в этом спектре наблюдаются на фоне производной магнитосопротивления, описанной нами ранее [10]. Для выделения линий резонансного спектра была найдена подгоночная аналитическая зависимость магнитосопротивления от поля в виде многочлена, и затем эта зависимость вычиталась из полной зависимости производной магнитосопротивления от поля.

После этого в спектре производной магнитопоглощения остаются резонансные линии, источником которых являются неспаренные спины различного происхождения. Рассмотрим их более подробно. Наиболее интенсивной является линия, обозначенная нами как $a_{0}$, которую мы приписываем резонансу электронов, находящихся в вырожденной зоне проводимости. Рядом, в несколько более сильном поле, заметна относительно слабая линия $a_{1}$, также, по-видимому, принадлежащая спинам в зоне проводимости. При дальнейшем увеличении поля в спектре проявляются более широкие и более слабые линии $b_{1}$ и $b_{2}$, слабая узкая линия $c_{1}$, очень слабая линия $b_{3}$, которая почти теряется в шумах. Обсудим происхождение и характеристики каждой из этих линий.

\section{4. Анализ линий ЭПР в слабых полях}

Обсуждение начнем с наиболее интенсивной линии $a_{0}$. Она имеет $g$-фактор $g=-14.14$, что довольно близко к значению $g=-13$, известному из литературы для концентрации электронов $n_{s}=1 \cdot 10^{18} \mathrm{~cm}^{-3}$ [3]. Большое значение $g$-фактора согласуется с малой эффективной массой электронов в этом материале. Одна из причин такого расхождения в значениях $g$-фактора может состоять в изменении эффективной массы электронов в зоне проводимости при изменении концентрации электронов. Однако концентрация электронов в исследованном нами образце в $\sim 8$ раз больше, чем в изученном в [3]. Такое различие должно приводить к увеличению эффективной массы, которая, как известно, в узкозонных полупроводниках растет с увеличением концентрации электронов [6]. Как следует из формул (1), рост эффективной массы должен приводить к уменьшению $g$-фактора. Эксперимент показывает, что в нашем случае происходит его увеличение.

Возможно, что наблюдаемый сдвиг $g$-фактора происходит из-за легирования материала примесью железа. Действительно, как показано в [7,12-14], легирование кристаллов $\mathrm{HgSe}$ примесью $\mathrm{Fe}$ приводит к появлению слабой намагниченности и внутреннего поля, которое 


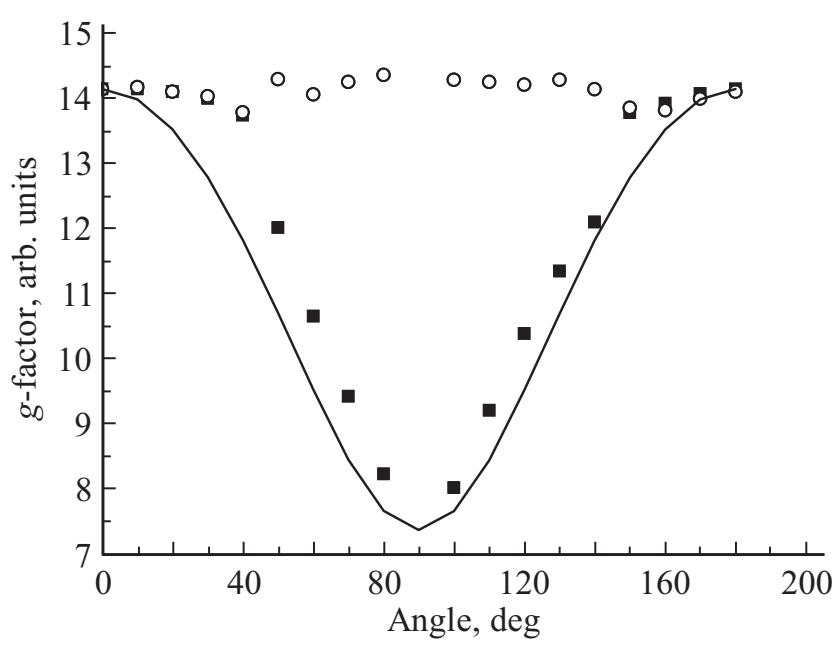

Pис. 2. Зависимость $g$-фактора свободных спинов от направления магнитного поля: $0^{\circ}-[001], 90^{\circ}-[011] ; T=3 \mathrm{~K}$. Заполненные точки отражают поведение резонансной линии, смещающейся при повороте, пустые точки отражают поведение неподвижной резонансной линии, линия на рисунке соответствует формуле (2).

сдвигает резонансное поле в область более слабых полей. Исходя из стандартных соотношений для частоты резонанса

$$
h v=g_{1} \beta H_{1}=g_{2} \beta H_{2},
$$

где $g_{1}$ и $g_{2}$ - значения $g$-фактора при наличии и отсутствии внутренних полей соответственно, $H_{1}$ и $H_{2}-$ значения резонансных полей, $\beta$ - магнетон Бора.

Отсюда определим величину резонансного поля в HgSe без примеси железа:

$$
H_{2}=H_{1} g_{1} / g_{2} \text {. }
$$

Это позволяет определить величину внутреннего поля

$$
\Delta H=H_{2}-H_{1}=509.9-468.8=41.1 \ni \text {. }
$$

Таким образом, можно предположить, что при легировании примесью железа за счет взаимодействия ионов $\mathrm{Fe}$ возникает слабая намагниченность. Связанное с ней внутреннее поле направлено навстречу внешнему и сдвигает резонансные линии в слабые поля.

Вторая особенность этой линии состоит в ее зависимости от направления поля. На рис. 2 показана зависимость $g$-фактора. Образец поворачивался вокруг оси [110] в плоскости (110). Эта плоскость содержит все главные оси симметрии кубического кристалла. Из рисунка видно, что $g$-фактор изменяется от 14.14 при направлении поля вдоль [001] до 7.76 при направлении поля вдоль направления $[0 \overline{1} 1]$. Кроме того, при повороте образца в плоскости (110) наблюдается более слабая неподвижная резонансная линия. По-видимому, она связана с перпендикулярным к плоскости поворота направлением [110]. Таким образом, главными осями тензора $g$ являются два направления [1̄ㅣ и и одно [100]. Интересно, что направление [111] никак не выделяется на рис. 2.

Как видно из рисунка, $g$-фактор изменяется с направлением поля и его величина при любом направлении в плоскости (110) может быть описана соотношением [2]

$$
g_{\mathrm{eff}}^{2}=g_{[001]}^{2} \cos ^{2} \varphi+g_{[0 \overline{1} 1]}^{2} \sin ^{2} \varphi,
$$

где $\varphi-$ угол между [001] и направлением магнитного поля $H$.

Как отмечено выше (формулы $(1 a)$ и $(16)$, в случае малых эффективных масс $g$-фактор достаточно простыми соотношениями связан с величиной эффективной массы.

Отсюда, пренебрегая единицей в скобках, получаем отношение эффективных масс:

$$
m_{l} / m_{t}=\left(g_{[001]}-2\right) /\left(g_{[0 \overline{1} 1]}-2\right) \approx 2.1 .
$$

Этот результат показывает, что зона проводимости в $\mathrm{HgSe}$ является не только непараболической (значение эффективной массы зависит от положения уровня Ферми), но и несферической (значение эффективной массы зависит от направления). Наибольшее значение эффективной массы наблюдается в направлениях [110].

Рядом с линией $a_{0}$ проявляется слабая узкая линия $a_{1}$. Ширина ее примерно такая же, как и линии $a_{0}$. По-видимому, она принадлежит электронам в зоне проводимости, обладающим большей эффективной массой. Ee $g$-фактор $g=-11$. Откуда в соответствии с формулой (6) отношение эффективных масс $m_{1} / m_{0} \approx 1.3$. К сожалению, линия проявляется только при одном направлении поля, и делать какие-либо выводы о ее происхождении преждевременно. Однако можно предположить, что слабый сдвиг $g$-фактора в сильные поля может быть связан с тем, что эти спины находятся в поле примеси железа и поэтому имеют другую эффективную массу и подвижность, как это показано в [12-14].

Объяснить такое увеличение эффективной массы можно на основе применения теории резонансного рассеяния в рамках подхода Фриделя [13]. Основа подхода заключается в том, что в зоне проводимости находятся два типа носителей заряда. Один из них - это собственные носители заряда, существующие независимо от концентрации примесных центров, а второй вносится примесями. Эти последние испытывают дополнительное рассеяние на примесных центрах, поскольку они слабо локализованы вблизи примесных центров. В [14] показано, что такая локализация должна проявляться и в магнитных свойствах полупроводника. К сожалению, малая амплитуда линии $a_{1}$ не позволяет более подробно рассмотреть влияние резонансного рассеяния в зоне проводимости на другие свойства, извлекаемые из анализа спектра ЭПР. Для этой цели необходимо найти способы усиления сигнала.

На рис. 1 показаны три линии, обозначенные как $b_{1}$, $b_{2}$ и $b_{3}$. Из него видно, что эти три линии вместе с 
линией $a_{0}$ составляют единый комплекс, в котором линии расположены на одинаковом расстоянии друг от друга. Действительно, резонансные поля: $H_{a 0}=0.46876$ кЭ; $H_{b 1}=1.03908$ кЭ; $H_{b 2}=1.53127$ кЭ; $H_{b 3}=2.03323$ кЭ, т. е. расстояние между ними $\Delta H \approx 0.5$ кЭ. Такое расположение линий, как показано в [15], является следствием мультиквантовых переходов. Такие переходы более вероятны в полупроводниках с большим $g$-фактором, когда ЭПР наблюдается в относительно слабых полях. При этом резонансные поля для мультиквантовых переходов становятся достижимыми. В нашем случае наряду с основной резонансной линией $a_{0}$ наблюдались двухквантовый переход $b_{1}$, трехквантовый переход $b_{2}$ и даже намеки на четырехквантовый переход $b_{3}$.

Причиной появления этого эффекта является большое время релаксации спина, обусловленное слабой связью с решеткой. Хотя конкретный механизм спиновой релаксации электронов проводимости в $\mathrm{HgSe}$ до настоящего времени не известен, ясно, что время спин-решеточной релаксации гораздо больше, чем рассеяние импульса и энергии электронов. Об этом говорит ширина резонансной линии:

$$
T_{1}=h /(g \beta \Delta H),
$$

где $h-$ постоянная Планка, $\Delta H-$ полуширина линии ЭПР, $\beta$ - магнетон Бора.

Из (7) следует, что при экспериментально наблюдаемой полуширине линии $\Delta H_{p p} \approx 20 \ni T_{1}=1.6 \cdot 10^{-8} \mathrm{c}$. Такое время релаксации спина позволяет наблюдать мультиквантовые переходы при использованных нами мощностях микроволнового поля. Из рис. 1 следует, что времена релаксации спинов в виртуальных состояниях гораздо короче, чем в основном. В результате ширина линий двухквантовых и трехквантовых переходов на порядок больше, чем основной.

Еще одна особенность резонансных линий от нелокализованных электронов состоит в асимметрии производных резонансного поглощения. Обычно количественный анализ асимметрии проводят через отношение амплитуд крыльев резонансной линии $A / B$, где $A-$ амплитуда низкополевого крыла производной линии поглощения, а $B$ - амплитуда высокополевого крыла. В [15] указывается на то, что в зависимости от соотношения между временем диффузии через скин-слой и временем спин-решеточной релаксации изменяется отношение $A / B$. Когда электрон диффундирует через скин-слой, много раз меняя направление спина, отношение $A / B \approx 2.7$. В противоположном пределе это отношение гораздо больше и может достигать 19.

Однако в нашем случае эксперимент дает $A / B=1.52$, что существенно меньше, чем предсказывает теория Дайсона [16]. Изучение ЭПР образцов компенсированного Ge:As вблизи фазового перехода изолятор-металл [9] показало, что отклонение от этой теории происходит в образцах с относительно большим сопротивлением, когда происходит переход формы линии от дайсоновской к лоренцевой. В $\mathrm{Ge}$ это происходит в области сопротивлений, когда толщина скин-слоя существенно больше толщины образца.

Другое предположение состоит в том, что время релаксации спина зависит от мощности микроволнового поля. Действительно, с увеличением этого поля сближаются концентрации спинов, направленных по и против постоянного магнитного поля. Это равносильно увеличению времени релаксации спина, т.е. время спин-решеточной релаксации начинает зависеть от мощности. В результате форма линии приближается к лоренцевой, что и наблюдается в эксперименте.

В работе [15] замечено, что отношение $A / B$ может служить характеристикой происхождения эффекта. Для электронов проводимости всегда $A / B>2.7$, а для электронов на парамагнитных примесях $A / B<2$.7. Но в нашем случае все электроны находятся в зоне проводимости, и для них в своей основе должна быть справедлива теория Дайсона.

В спектре ЭПР поглощения наблюдается еще одна одиночная линия $c_{1}$. Ее форма и положение заметно отличаются от обсуждавшийся ранее линии поглощения, обусловленной свободными электронами в зоне проводимости.

Анализ литературы $[15,17]$ показывает, что наиболее вероятным источником этой линии являются ионы $\mathrm{Fe}^{+}$, которые дают резонансную линию при $g \approx 4$. Хорошо известно, что основным состоянием примеси железа в $\mathrm{HgSe}$ является состояние $\mathrm{Fe}^{3+}$ [7], в котором находятся эти атомы, замещая в матрице атомы Hg. Можно полагать, что ионы $\mathrm{Fe}^{+}$находятся в междоузлиях. Таких состояний мало, но благодаря тому, что их спектр ЭПР кардинально отличается от спектров ионов $\mathrm{Fe}^{3+}$, эти атомы можно изучать таким методом. Однако более глубокое изучение спектров $\mathrm{Fe}$ в $\mathrm{HgSe}$ не входит в задачу данной работы.

\section{5. Заключение}

В работе впервые описаны ЭПР спектры электронов в зоне проводимости в кристаллах $\mathrm{HgSe}: \mathrm{Fe}$, полученные прямыми измерениями микроволнового резонансного поглощения. Их анализ позволяет выявить ряд новых эффектов и определить ряд параметров.

1. Определен $g$-фактор линии резонансного поглощения. Оказалось, что он зависит от направления внешнего магнитного поля. Это значит, что величина эффективной массы электронов в этом материале зависит от направления: $m_{l} / m_{t} \approx 2.1$.

2. Обнаружен сдвиг сигнала ЭПР в область более слабых полей по сравнению с литературными данными. Он объяснен как результат появления дополнительного внутреннего магнитного поля (слабой спонтанной намагниченности), которая создается ионами $\mathrm{Fe}^{3+}$.

3. В более сильных полях по сравнению с полем резонансной линии основных переходов $H=0.46876$ кЭ 
обнаружены мультиквантовые переходы: двухквантовые в поле $H=1.03908$ кЭ, трехквантовые в поле $H=1.53127$ кЭ и, возможно, четырехквантовые переходы в поле $H=2.03323$ кЭ.

4. Обнаружено, что искажение формы линии ЭПР отличается от предсказанной теорией Дайсона. Предполагается, что это происходит из-за зависимости времени спин-решеточной релаксации от мощности микроволнового поля.

Работа подготовлена при частичной поддержке Российского фонда фундаментальных исследований (проект 16-02-00352) и Программы президиума РАН 1.4. „Актуальные проблемы физика низких температур“.

Авторы благодарны П.В. Семенихину и Д.А. Фролову за помощь в проведении экспериментов и участие в обсуждении.

\section{Список литературы}

[1] Дж. Людвиг, Г. Вудбери. Электронный спиновый резонанс в полупроводниках (М., Мир, 1964).

[2] И.М. Цидильковский. УФН, 162, 63 (1992).

[3] L. Bliek, G. Landwehr. Phys. Status Solidi, 31, 115 (1969).

[4] А. Абрагам, Б. Блини. Электронный парамагнитный резонанс переходных ионов (М., Мир, 1972).

[5] Z. Wilamowski, A. Mycielski, W. Jantsch, G. Hendorfer. Phys. Rev. B, 38, 3621 (1988).

[6] A. Lenard, T. Dietl, M. Sawicki, W. Dobrovolski, K. Dybko, T. Skoskiewicz, W. Plesiewicz, S. Miotkowska, A. Witek. J. Low Temp. Phys., 80, 15 (1990).

[7] А.Е. Лончаков, В.И. Окулов, С.Ю. Паранчич. ФНТ, 35, 92 (2009).

[8] Ч. Пул. Техника ЭПР спектроскопии (М., Мир, 1970) [Пер. с англ.: С.P. Poole. Electron spin resonance (Int. Pub. N. Y.-London-Sydney, 1967)].

[9] А.И. Вейнгер, А.Г. Забродский, Т.В. Тиснек, С.И. Голощапов, П.В. Семенихин. ЖТФ, 83 (12), 103 (2013).

[10] А.И. Вейнгер, Т.В. Тиснек, И.В. Кочман, В.И. Окулов. ФТП, 51, 172 (2017).

[11] C.R. Whitsett. Phys. Rev., 138, A829 (1965).

[12] Г.А. Альшанский, Т.Е. Говоркова, В.И. Окулов, А.В. Королев, С.Ю. Паранчич. ФНТ, 34, 613 (2008).

[13] В.И. Окулов, Л.Д. Сабирзянова, К.С. Сазонова, С.Ю. Паранчич. ФНТ, 30, 441 (2004).

[14] В.И. Окулов, Е.А. Памятных, Г.А. Альшанский. ФНТ, 35, 194 (2009).

[15] С.А. Альтшулер, Б.М. Козырев. Электронный парамагнитный резонанс соединений элементов промехуточных групn (М., Наука, 1972).

[16] F.J. Dyson. Phys. Rev., 98, 349 (1955).

[17] G. Feher, A.F. Kip. Phys. Rev., 98, 337 (1955).

Редактор Г.А. Оганесян

\section{Spin resonance of conduction electrons in the $\mathrm{HgSe}$}

\author{
A.I. Veinger ${ }^{1}$, I.V. Kochman ${ }^{1}$, V.I. Okulov ${ }^{2}$, \\ M.D. Andriichuk ${ }^{3}$, L.D. Paranchich ${ }^{3}$ \\ ${ }^{1}$ loffe Institute, \\ 194021 St. Petersburg, Russia \\ ${ }^{2}$ Miheev Institute of Metal Physics \\ of Ural Branch of Russian Academy of Sciences, \\ 620137 Ekaterinburg, Russia \\ ${ }^{3}$ Chernovtsy National University, \\ 48012 Chernovtsy, Ukraine
}

Abstract Gapless semiconductor $\mathrm{HgSe}$ with a different $\mathrm{Fe}$ doping concentration was investigated. HgSe:Fe samples were studied by the means of electron spin resonance technique. It was found that unpaired spins of different origin lead to the multiple resonance lines. These resonance lines were determined and analyzed. Hydrogen-like model was used to describe the properties of electrons localized on shallow impurities. The impact of internal field on the resonance lines was established. It was discovered that the conduction band of $\mathrm{HgSe}$ is nonspherical as well as a nonparabolic. 